\title{
CDISC Kidney Transplant Therapeutic Area User Guide Version 1.0
}

National Cancer Institute

\section{Source}

National Cancer Institute. CDISC Kidney Transplant Therapeutic Area User Guide Version

1.0. NCl Thesaurus. Code C161445.

The 1.0 version of the CDISC Kidney Transplant therapeutic area user guide. 